\title{
Sentido de vida, dependência funcional e qualidade de vida em idosos
}

\author{
Meaning in life, functional dependence and quality of life among the elderly
}

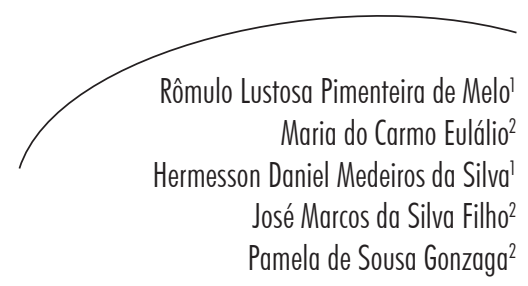

\section{Resumo}

Este estudo objetivou avaliar os índices de sentido de vida (SV), qualidade de vida (QV) e dependência funcional (DF) de idosos, e observar se o SV poderia atuar como recurso protetor capaz de diminuir os efeitos da DF na QV. Caracteriza-se por ser transversal, de base domiciliar, com 210 participantes que possuem média de idade de 74 anos $(d p=7,70)$, a maioria do sexo feminino $(68,4 \%)$ e com renda média de 868 reais $(d p=1,100)$. Os instrumentos utilizados foram: Mini-Exame do Estado Mental, medida de independência funcional, questionário de qualidade de vida para idosos, teste de propósito de vida e um questionário sociodemográfico. Os resultados indicaram que a maioria da amostra apresentou altos índices de QV, SV e baixos índices de DF, além disso, a relação entre DF e QV para os idosos com alto SV não foi significativa $[\beta=$ $-0,19 ; t(93)=-1,92 ; p>0,05]$. Portanto, a QV dos idosos que apresentaram níveis altos de SV se torna mais protegida contra as alterações dos níveis de DF. Sendo assim, o sentido de vida atuou como recurso psicológico protetor.

\section{Abstract}

This study aimed to evaluate the meaning of life indices (SV), quality of life (QOL) and functional dependency (FD) of the elderly, and observe whether the SV could act as protective feature capable of reduce the effects of DF on QOL. It is characterized by being cross-sectional of domiciliary basis, with 210 participants who have an average age of 74 years $(\mathrm{SD}=7.70)$, mostly female $(68.4 \%)$ and with an average income of 868 reais $(\mathrm{sd}=1.100)$. The instruments used were: Mini-mental State Examination, functional independence measure, quality of life questionnaire for seniors, life purpose test and a socio-demographic questionnaire. The results indicated that the majority of the sample reported higher QOL, SV and low rates of

Palavras-chave: Idoso. Saúde do Idoso. Vida. Sentido de Vida. Idoso Fragilizado. Qualidade de Vida.
Key words: Elderly. Health of the Elderly. Life. Meaning of Life. Frail Elderly. Quality of Life.

Programa de Pós-graduação em Psicologia Social. Universidade Federal da Paraíba. João Pessoa, PB. Brasil.

2 Departamento de Psicologia, Centro de Ciências Biológicas e da Saúde. Universidade Estadual da Paraíba. Campina Grande, PB, Brasil.

Órgãos financiadores: Programa de Incentivo à Pós-graduação e Pesquisa da Universidade Estadual da Paraíba - PROPESQ/PIBIC/UEPB/CNPq. 
DF; moreover, the relationship between DF and QL for seniors with high SV was not significant $[\beta=-0.19, \mathrm{t}(93)=-1.92, \mathrm{p}>0.05]$. Therefore, QOL of elderly people with high levels of SV became more protected against changes in the levels of DF.

Thus, the SV acted as a psychological resource protector.

\section{INTRODUÇÃo}

A população idosa tem apresentado as maiores taxas de crescimento. Segundo a Organização Mundial da Saúde (OMS), a estimativa é que até 2025 existam cerca 1,2 bilhões de pessoas com idade igual ou superior a 60 anos, o que representará um quinto da população mundial. ${ }^{1}$ Dados do último censo realizado pelo Instituto Brasileiro de Geografia e Estatística (IBGE) apontam que dos 185,7 milhões de brasileiros, aproximadamente $10 \%$ são idosos. ${ }^{2}$ A conquista da maior longevidade, no entanto, pode ser acompanhada por uma série de dificuldades que podem interferir negativamente na qualidade de vida $(\mathrm{QV})$ dessa população.

Dentre essas dificuldades, destaca-se a dependência funcional (DF), isto é, a presença de dificuldade ou a necessidade de ajuda para realização de atividades que fazem parte do cotidiano, sejam elas básicas ou complexas, necessárias para uma vida independente na sociedade. ${ }^{3}$ Pesquisas indicam a relação da DF com aspectos ligados à saúde, tais como aumento de doenças crônicas, ${ }^{4,5}$ déficit $\operatorname{cognitivo~}^{6}$ e maiores níveis de estresse. ${ }^{7,8}$

O processo de envelhecimento tende a ser marcado por mudanças fisiológicas, psicológicas e sociais (declínio do funcionamento físico, aparecimento ou o agravamento de doenças somáticas, dificuldades com a memória, isolamento social), que podem proporcionar uma experiência estressante e diminuir a QV. ${ }^{8}$ Paschoal $^{9}$ relata que a realização satisfatória de atividades de vida diária estão entre os eventos da vida que podem interferir na QV, pois pode levar o idoso a sentimentos de frustração e dependência. Segundo o grupo de qualidade de vida da Organização Mundial da Saúde
(WHOQOL), a QV seria “a percepção do indivíduo de sua posição na vida, no contexto da cultura e sistema de valores nos quais ele vive e em relação aos seus objetivos, padrões e preocupações". ${ }^{10}$

Esse conceito evidencia o papel da percepção do sujeito no processo de avaliação de situações adversas. Diante das mesmas condições, o impacto dos estressores nos indivíduos difere, em parte, pela possibilidade do emprego de recursos psicossociais, que proporcionem estratégias de enfrentamento frente às situações aversivas. ${ }^{11}$ Dentre tais recursos, o sentido de vida (SV) pode ser considerado um fator capaz de auxiliar no enfrentamento de situações adversas e de potencializar os níveis de saúde e QV dos sujeitos. ${ }^{12-14}$

O SV há muito tempo é discutido pela filosofia, mas Viktor Frankl (1905-1997) foi o primeiro a abordar enquanto construto psicológico. Embora não tenha utilizado definições claras sobre o SV, Frankl sugere que o mesmo está relacionado à crença de que a vida do sujeito é plena de significados e que transcende a própria realidade material, está ligado a crença de um propósito, uma direção, razão particular para a existência. ${ }^{15,16}$

Para uma vida encontrar sentido, Frank $1^{15}$ sugere três categorias de valores: valor criativo que produz algo significativo, fazer uma boa ação; valor vivencial - são as experiências de trocas afetivas ou mesmo interagindo com os objetos do mundo, sentido que pode ser encontrado nas experiências da vida; valor atitudinal - é aquele que transforma as dores/tragédias pessoais em boas lições que levará ao crescimento.

Diante disso, ao considerar que a DF parece afetar a QV dos idosos, o presente trabalho possui 
como objetivo examinar os índices de sentido de vida (SV), qualidade de vida (QV) e dependência funcional (DF) de idosos, e observar se o SV poderia atuar como recurso protetor capaz de diminuir os efeitos da DF na QV.

\section{MÉTODO}

Este estudo se caracteriza por ser transversal, de base domiciliar, e faz parte de uma pesquisa maior intitulada "Perfil de Funcionalidade e Fragilidade de Idosos da Cidade de Campina Grande-PB”, que utilizou a amostra da Rede FIBRA* para fins de comparação. Assim, tomouse como referência uma amostra composta por 254 idosos, os quais apresentaram desempenho cognitivo acima da nota de corte ajustada para escolaridade no Mini-Exame do Estado Mental (MEEM). Após dois anos deste estudo, foram encontrados os endereços de 229 idosos, dos quais seis já haviam falecido e 13 começaram a apresentar comprometimento cognitivo. Neste sentido, já não poderiam fazer parte do estudo. A amostra final, portanto, foi composta por 210 idosos da zona urbana de Campina Grande-PB, com média de idade de 74 anos ( $\mathrm{dp}=7,7)$. Destes, $68,4 \%$ eram mulheres. Quanto ao estado civil, 47\% declararam ser casados ou vivendo maritalmente e $37,2 \%$ declararam ser viúvos(as). Quanto ao nível de escolaridade, a maioria dos idosos tinha até o ensino básico completo, sendo que $27,4 \%$ não eram escolarizados. A média da renda foi de 868 reais $(\mathrm{dp}=1,100)$, com uma moda de $515(55,15 \mathrm{da}$ amostra) (salário mínimo vigente de $\mathrm{R} \$ 515,00$ ).

\section{Procedimentos}

A pesquisa foi realizada após aprovação do Comitê de Ética em Pesquisa da Universidade

\footnotetext{
* Para o estudo da Rede Fibra (acrônimo de fragilidade em idosos brasileiros), os idosos foram recrutados por meio de setores censitários urbanos, sendo sorteados ao acaso de acordo com a lista fornecida pelo IBGE. O cálculo amostral foi realizado para uma prevalência de $50 \%$, com um limite de confiança de 1,96. O coeficiente de correção amostral foi de 2,1, uma vez que a amostra foi por conglomerado, e a precisão aceita para a prevalência estimada foi de $A=5 \%$
}

Estadual da Paraíba (CEP/UEPB - protocolo $\mathrm{n}^{\mathrm{o}}$ 0022.0.133.000-10). A coleta de dados ocorreu entre os meses de junho e dezembro de 2010, seguindo os critérios éticos postulados pela Resolução no 196/96 do Conselho Nacional de Saúde.

Os recrutadores foram compostos por oito estudantes devidamente treinados do curso de graduação de Psicologia da UEPB. Eles se dividiram em quatro duplas e abordavam os idosos em suas residências, com base no banco de dados da pesquisa anterior da Rede Fibra.

Aos idosos eram explicados os objetivos do estudo, apresentando-lhes o Termo de Consentimento Livre e Esclarecido (TCLE), juntamente com os questionários a serem respondidos. Em caso de aceite de participação do estudo e após a assinatura do TCLE, os idosos eram submetidos à avaliação de seu status cognitivo mediante um teste de rastreio cognitivo (Mini-Exame do Estado Mental - MEEM, validado por Folstein, Folstein \& Mc Hugh). ${ }^{17}$ Os idosos que pontuaram abaixo da nota de corte para seu nível de escolaridade foram excluídos, medida necessária para maior confiabilidade das respostas. Foram oferecidas explicações compatíveis com o respeito à dignidade dos sujeitos. A coleta de dados foi realizada com duração média de 50 minutos.

\section{Descrição dos instrumentos psicométricos}

O Mini-Exame do Estado Mental (MEEM) consiste num questionário de 30 itens e avalia sete categorias de funções cognitivas. Sua pontuação total pode atingir 30 pontos, assim distribuídos: orientação temporal, orientação espacial, memória imediata, atenção e cálculo, evocação atrasada de palavras, linguagem e praxia construtiva. ${ }^{17}$ As notas de corte utilizadas para exclusão pelo MEEM foram baseadas em Brucki et $a .^{18}$ Nesta pesquisa, o MEEM foi utilizado como critério de exclusão, proporcionando maior confiabilidade nas respostas dadas aos questionários. 


\section{Questionário da qualidade de vida para idosos (WHOQOL-OLD)}

Foi desenvolvido pelo grupo de qualidade de vida da Organização Mundial da Saúde (OMS), ${ }^{19}$ com o objetivo de mensurar a satisfação do indivíduo com sua vida e sua percepção a respeito da influência que as doenças causam em sua vida. Este questionário consta de 24 itens com resposta tipo Likert de 1 a 5, divididos em seis facetas. Cada faceta é composta por quatro itens, assumindo uma pontuação padronizada de 0 a 100. Os itens das facetas, somados, geram um escore overall (geral). Os domínios são: autonomia; funcionamento dos sentidos; atividades passadas, presentes e futuras; participação social; morte e morrer; e intimidade.

Quadro 1 - Conteúdos/Conceitos das facetas do WHOQOL-OLD.

\begin{tabular}{|ll|}
\hline \multicolumn{1}{|c|}{ Faceta } & \multicolumn{1}{c|}{ Conteúdo/Conceito } \\
\hline Autonomia & $\begin{array}{l}\text { Independência do idoso; ser capaz e livre de viver de modo } \\
\text { autônomo e de tomar suas próprias decisões. }\end{array}$ \\
Funcionamento dos sentidos & $\begin{array}{l}\text { Impacto da perda dos funcionamentos dos sentidos na } \\
\text { qualidade de vida. }\end{array}$ \\
Atividades passadas, presentes e futuras & $\begin{array}{l}\text { Satisfação com as realizações na vida, e com objetivos a } \\
\text { serem alcançados. }\end{array}$ \\
Participação social & $\begin{array}{l}\text { Participação em atividades da vida social, especialmente as } \\
\text { ligadas à comunidade. } \\
\text { Morte e morrer }\end{array}$ \\
Preocupações e medos acerca da morte e morrer. \\
Ser capaz de ter relacionamentos íntimos e pessoais.
\end{tabular}

Fonte: Chachamovich. ${ }^{20}$

\section{Teste de propósito de vida (PIL-Test 12)}

Originalmente desenvolvido por Crumbaugh \& Maholick ${ }^{21}$ faz uma avaliação da sensação de realização existencial experienciada pelo sujeito. O instrumento original é composto por 20 itens e é validado em diversos países, mantendo bons índices de consistência interna, com Alfas de Cronbach variando, em geral, de 0,70 a $0,90 .^{14,22}$ A versão utilizada neste estudo refere-se a uma adaptação do instrumento original, escala reduzida composta de 12 itens. ${ }^{23} \mathrm{O}$ questionário possui resposta em formato tipo Likert variando de 1 (discordo totalmente) a 7 (concordo totalmente).

\section{Medida de independência funcional (MIF)}

É um instrumento que avalia a independência funcional por meio do desempenho de cada indivíduo nas suas atividades do dia a dia. Foi desenvolvida na década de 1980 pela Academia Americana de Medicina de Reabilitação e validada para a população de idosos por Pollak et al. $^{24} \mathrm{~A}$ escala possui sua reprodutibilidade e validação para a realidade brasileira realizada por Riberto et al. ${ }^{25}$ Consiste em 18 tarefas, referentes às subescalas de autocuidado, controle esfincteriano, mobilidade/transferência, locomoção, comunicação e cognição social. Cada 
item é classificado em uma escala de graus de dependência de sete níveis, sendo o valor zero correspondente à dependência total e o valor sete correspondente a independente - sendo assim, quanto menor a pontuação na escala, maior o grau de DF. ${ }^{14}$ É dividida em MIF motora, que envolve as dimensões autocuidado, controle esfincteriano, transferência e locomoção; e MIF cognitiva que engloba as de comunicação e cognição social. No presente estudo, entretanto, utilizou-se apenas da MIF motora na medida em que se aplicou para a avaliação cognitiva.

Por fim, para categorização sociodemográfica foi construído um questionário com perguntas relacionadas à: idade, sexo, estado civil, grau de escolaridade e renda mensal do idoso.

Análise dos dados

Utilizou-se o PASW (versão 19) para tabulação e análise dos dados. Após a descrição dos dados sociodemográficos (com medidas de tendência central e dispersão), checaram-se os parâmetros psicométricos dos instrumentos de qualidade e sentido de vida (análise dos componentes principais e Alpha de (ronbach). Em seguida, foram averiguadas as medidas descritivas dos instrumentos de Whoqol-Old, Pil-Test 12 e a MIF. Posteriormente, com a finalidade de responder se o SV poderia atuar como recurso protetor capaz de diminuir os efeitos da Dependência Funcional (DF) na QV, foi realizada uma análise de moderação seguindo os passos sugeridos por Baron \& Kenny. ${ }^{26}$ Porém, antes do inicio dessa análise, as pontuações da MIF foram multiplicados por -1, para que passassem a representar não mais a Independência Funcional, mas a Dependência Funcional (DF). Sendo assim, realizou-se correlações de Pearson para verificar as relações entre os construtos de SV, IF e QV; posteriormente, foram feitas análises de regressão linear, para entender se o SV poderia moderar a relação da IF com a QV. O erro aceito para todas as medidas é de 5\%, ou seja, $p \leq 0,05$.

\section{RESULTADOS}

Propriedades psicométricas das medidas de qualidade vida e sentido de vida

Ambas as matrizes de correlações dos instrumentos WHOQOL-OLD e PIL-Test 12 revelam a possibilidade de fatorialização. O WHOQOL-OLD apresentou teste de esfericidade de Bartlett $\mathrm{x}^{2}=1413,00$ e KMO = $0,79, \operatorname{com} p<0,001$. No que se refere à estrutura, não foram realizadas análises exploratórias, pois neste caso, seguindo recomendações da OMS, os fatores foram fixados seguindo as instruções da própria escala. ${ }^{19}$ Os índices de consistência interna (Alfas de Cronbach, $\alpha$ ) se mostraram adequados para propósitos de pesquisa, com exceção da faceta "autonomia" $(\alpha=0,48)$, como seguem: "funcionamento dos sentidos" $(\alpha=$ $0,81)$, "atividades passadas, presentes e futuras" $(\alpha$ $=0,61)$, "participação social" $(\alpha=0,66)$, "morte e morrer" $(\alpha=0,73)$ e "intimidade" $(\alpha=0,72)$; o "fator geral de qualidade de vida" também apresentou adequação deste parâmetro $(\alpha=0,81)$.

Para a escala PIL-Test 12, o índice de KMO $(0,80)$ e o teste de esfericidade de Bartlett $\left(x^{2}=447,181, p<0,001\right)$ apresentam resultados satisfatórios. Por meio de análise fatorial exploratória (método de extração de componentes principais - PC, e rotação varimax), foram encontrados dois fatores, nomeados de vazio existencial e realização existencial, ambos com eigenvalue $>1$ que explicam conjuntamente $39,8 \%$ da variância total. Os índices de consistência interna foram, respectivamente: $\alpha=$ 0,$73 ; \alpha=0,63$. Um fator geral incluindo todos os itens do instrumento também apresentou boa consistência interna $(\alpha=0,77)$, sendo uma opção viável de avaliação. Para este fator geral, deu-se o nome de "sentido de vida". 
Medidas descritivas das escalas de qualidade de vida, sentido de vida e medida de independência funcional

Os resultados encontrados em cada uma das escalas utilizadas são descritos na tabela 1. No instrumento de QV para idosos (Whoqol-Old), o domínio "funcionamento dos sentidos" foi o que apresentou maior média $(\mathrm{m}=75,91 ; \mathrm{dp}=20,83)$, seguido pelos domínios "morte e morrer" $(\mathrm{m}=67,97 ; \mathrm{dp}=23,49)$, “intimidade" $(\mathrm{m}=67,62$; $\mathrm{dp}=16,74)$ e "participação social" ( $\mathrm{m}=65,93$; $\mathrm{dp}=16)$. O domínio "morte e morrer" foi o que apresentou maior desvio-padrão, evidenciando a maior variação em torno da média. As facetas que apresentaram as menores médias foram, respectivamente, "autonomia" ( $\mathrm{m}=60,14$; $\mathrm{dp}=16,35)$ e "atividades passadas, presentes e futuras" ( $\mathrm{m}=64,71 ; \mathrm{dp}=15,91)$. A QV geral apresentou média de 66,95 (dp=10,98).

$\mathrm{Na}$ escala de SV, a média encontrada para o fator geral (sentido de vida) foi de 5,62 (dp= 0,89) e as de realização existencial e vazio existencial foram, respectivamente, $(\mathrm{m}=5,9 ; \mathrm{dp}=0,87)$ e $(\mathrm{m}=2,3 ; \mathrm{dp}=1,0)$.

Quanto às medidas de independência funcional, o fator geral apresentou média de 84,13 ( $\mathrm{dp}=5,89$ ). Constatou-se que 95,7\% dos idosos apresentaram escores entre 75 e 91 pontos, correspondente à classificação independente, e $4,3 \%$ necessitavam de assistência em até $50 \%$ das atividades diárias.

Tabela 1 - Descrição dos escores do Whoqol-Old, Pil-Test 12 e da MIF. Campina Grande-PB, 2010

\begin{tabular}{lcccc}
\hline \multicolumn{1}{c}{ Domínios do Whoqol-Old } & N & Média & DP & Intervalo \\
\hline Funcionamento dos sentidos & 210 & 75,91 & 20,83 & $0-100$ \\
Morte e morrer & 210 & 67,97 & 23,49 & $0-100$ \\
Intimidade & 210 & 67,62 & 16,74 & $0-100$ \\
Participação social & 210 & 65,93 & 16,00 & $0-100$ \\
Autonomia & 210 & 60,14 & 16,35 & $0-100$ \\
Atividades (pas., pres.,fut.) & 210 & 64,71 & 15,91 & $0-100$ \\
QV - geral & 210 & 66,95 & 10,98 & $0-100$ \\
\hline Fatores do Pil-Test 12 & $\mathbf{N}$ & Média & DP & Intervalo \\
\hline Sentido de vida & 210 & 5,62 & 0,89 & $1-7$ \\
Realização existencial & 210 & 5,90 & 0,87 & $1-7$ \\
Vazio existencial & 210 & 2,31 & 2,15 & $1-7$ \\
\hline Dimensões da MIF & $\mathbf{N}$ & Média & DP & Intervalo \\
\hline Autocuidados & 210 & 6,80 & 0,41 & $1-7$ \\
Controle dos esfíncteres & 210 & 6,68 & 0,56 & $1-7$ \\
Mobilidade & 210 & 6,78 & 1,01 & $1-7$ \\
Locomoção & 210 & 4,78 & 0,26 & $1-7$ \\
MIF motora total & 210 & 6,26 & 0,89 & $1-7$ \\
\hline
\end{tabular}


Correlações entre qualidade de vida, sentido de vida e independência funcional

A magnitude e a direção das associações do DF e SV com a qualidade de vida dos idosos foram investigadas por meio da análise de correlação de Pearson (r). Os resultados são apresentados na tabela 2.
A faceta "atividades passadas, presentes e futuras" e o escore geral de QV apresentaram as correlações mais fortes com os fatores do Pil-Test 12. Para as dimensões da MIF, a faceta "funcionamento dos sentidos" e a QV geral apresentaram as maiores correlações.

Tabela 2 - Correlações da incapacidade funcional e sentido de vida com os domínios da qualidade de vida. Campina Grande-PB, 2010.

\begin{tabular}{|c|c|c|c|c|c|c|c|}
\hline & $\begin{array}{c}\text { Func. dos } \\
\text { Sentidos }\end{array}$ & $\begin{array}{l}\text { Morte } \\
\text { Morrer }\end{array}$ & Intimidade & $\begin{array}{l}\text { Part. } \\
\text { Social }\end{array}$ & Autonomia & $\begin{array}{l}\text { Ativ.(pas., } \\
\text { pres.,fut.,) }\end{array}$ & QV Geral \\
\hline SV geral & $0,39 * *$ & $0,18^{*}$ & $0,34 * *$ & $0,25 * *$ & $0,25^{* *}$ & $0,45^{* *}$ & $0,45^{* *}$ \\
\hline $\begin{array}{l}\text { Vazio } \\
\text { existencial }\end{array}$ & $-0,35^{* *}$ & 0,11 & $-0,33^{* *}$ & $-0,27^{* *}$ & $-0,27^{* *}$ & $-0,38^{* *}$ & $-0,44^{* *}$ \\
\hline $\begin{array}{l}\text { Realização } \\
\text { existencial }\end{array}$ & $0,33^{* *}$ & 0,12 & $0,18^{*}$ & $0,14 * *$ & $0,15^{*}$ & $0,40 * *$ & $0,37^{* *}$ \\
\hline MIF geral & $0,41 * *$ & 0,14 & 0,12 & $0,21 * *$ & $0,23 * *$ & $0,25^{* *}$ & $0,33^{* *}$ \\
\hline Auto-Cuidado & $0,37 * *$ & 0,08 & 0,01 & $0,17 *$ & $0,25^{* *}$ & $0,28^{*}$ & $0,34^{* *}$ \\
\hline $\begin{array}{l}\text { Contr. dos } \\
\text { esfíncteres }\end{array}$ & $0,24 * *$ & 0,11 & 0,03 & $0,19 * *$ & $16^{* *}$ & $0,14^{* *}$ & $25^{* *}$ \\
\hline Mobilidade & $0,30^{* *}$ & 0,11 & 0,06 & $0,14^{*}$ & $0,25^{* *}$ & $0,22 * *$ & $0,30^{* *}$ \\
\hline Locomoção & $0,26 * *$ & 0,05 & 0,08 & $0,20 * *$ & 0,09 & $0,22 * *$ & $0,25^{* *}$ \\
\hline
\end{tabular}

* Correlações com significância $\leq 0,05 . * *$ Correlações com significância $\leq 0,01$.

Sentido de vida como moderador do efeito da dependência funcional na qualidade de vida

Para verificar se o SV seria capaz de minimizar os efeitos da dependência funcional na QV geral dos idosos, as análises foram divididas em dois passos: $1^{\circ}$ passo - verificar se o SV e a dependência funcional DF explicariam a QV geral; em caso afirmativo, o $2^{\circ}$ passo teria como objetivo averiguar se a predição da DF na QV geral se alteraria na presença de níveis diferentes de SV (alto SV - baixo SV).

\section{$1^{\circ}$ Passo}

Foram realizadas duas regressões lineares simples; a primeira, tendo como preditor o SV e a segunda tendo como preditor a DF, ambas tendo como variável dependente a QV geral (tabela 3). 
Tabela 3 - Regressões lineares simples entre sentido de vida, incapacidade funcional e qualidade de vida geral. Campina Grande-PB, 2010.

\begin{tabular}{lccccccc}
\hline & \multicolumn{7}{c}{ Qualidade de Vida Geral (VD) } \\
\hline & B & SE & $\beta$ & Gl & $r^{2}$ & $\mathrm{t}$ & $p$ \\
Sentido de vida (VI) & 32,93 & 4,34 & 0,49 & 197 & 0,24 & 7,93 & $\geq 0,001$ \\
Dependência funcional (VI) & 10,02 & 1,70 & 0,41 & 169 & 0,17 & $-5,87$ & $\geq 0,001$ \\
\hline
\end{tabular}

$\mathrm{SE}=$ erro padrão; $\mathrm{VI}$ = variável independente; $\mathrm{VD}$ = variável dependente.

Os resultados mostraram que ambas as variáveis $\mathrm{SV}(\beta=0,49 ; t(197)=7,93 ; p<0,001)$ e DF $(\beta=-0,41 ; t(169)=-5,87 ; p<0,001)$ mostraram-se como preditoras de QV geral. O SV explicou $24 \%$ da variação da QV geral e a DF $17 \%$ da variação da QV geral.

\section{$2^{\circ}$ Passo}

Considerando a predição que a DF apresenta sobre o índice geral de QV, optou-se por averiguar se o SV seria capaz de diminuir esta relação. Neste sentido, primeiramente foi inserida a interação entre DF e SV (i.e., criou-se um termo de interação ao multiplicar a DF pelo SV), com os resultados demonstrando que esta interação foi um preditor significativo da pontuação total em qualidade de vida $[\beta=-0,352 ; t(154)=-4,65, p<0,001]$. Isso sugeriu um efeito moderador do $S V^{26}$.

Com o propósito de conhecer a direção da moderação previamente identificada (dividiramse os idosos em categorias segundo sua pontuação em SV). Dessa forma, dicotomizou-se a variável SV com base na mediana, ficando dois grupos de SV: aqueles que apresentaram baixo SV e aqueles que apresentaram alto SV. Prosseguiu-se com uma regressão linear simples para cada um destes grupos.

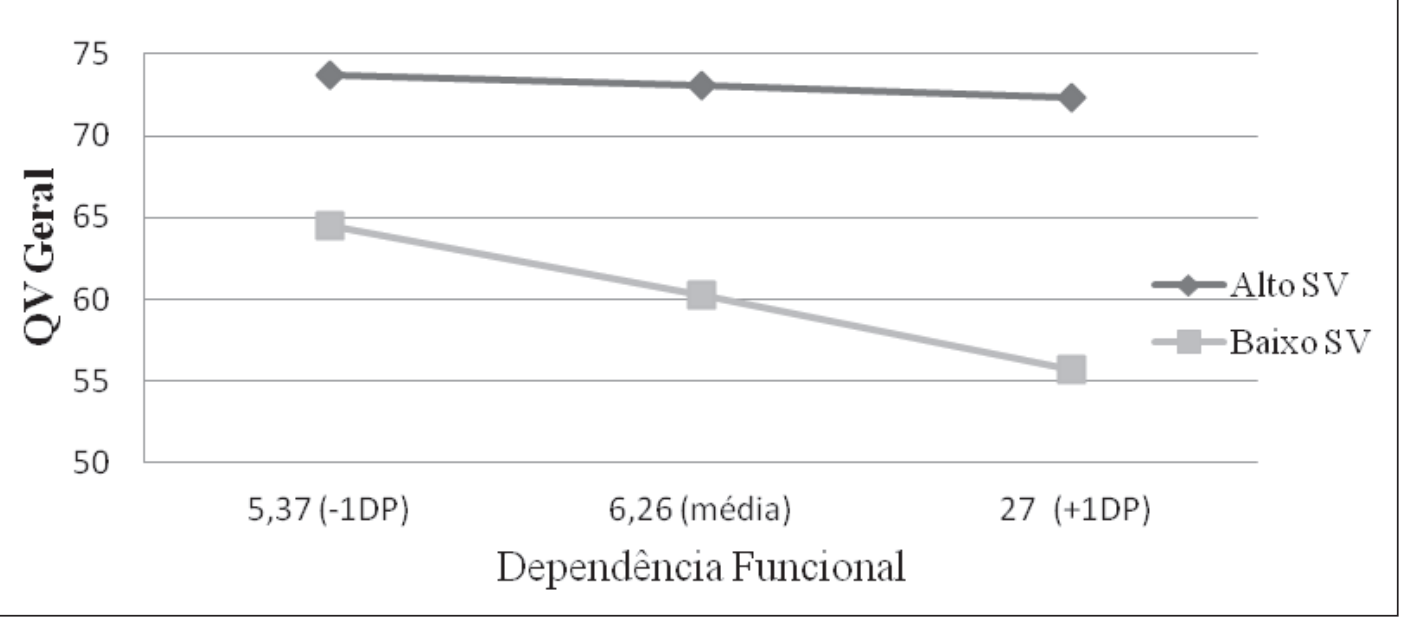

Figura 1 - Efeito moderador do SV na relação da DF com a QV Geral. 
Conforme mostra a figura 1, para os idosos que possuem níveis baixos de SV, a DF foi um preditor significativo da QV $[\beta=-0,46 ; t(73)=$ $-4,39 ; p<0,001]$; no entanto, para o grupo com alto $\mathrm{SV}$, o mesmo não foi observado $[\beta=-0,19$; $t(93)=-1,92 ; p>0,05])$. Assim, a QV geral dos idosos que apresentaram níveis altos de SV ficou mais protegida contra as alterações dos níveis de DF. O SV atuou como um recurso protetor da QV geral.

\section{DISCUSSÃO}

Medidas descritivas das escalas de qualidade de vida, sentido de vida e medida de independência funcional

Embora se observe crescente interesse dos pesquisadores na temática da $\mathrm{QV}$, não foram encontrados estudos no contexto brasileiro relacionados ao WHOQOL-Old com população geral de idosos na comunidade. Comumente, os que empregam este instrumento têm considerado populações específicas de idosos.

A respeito das dificuldades relacionadas às habilidades sensoriais do domínio "funcionamento dos sentidos" (Whoqol-Old), a maioria da população de idosos ${ }^{27}$ relata que muitas vezes estas não interferem na $\mathrm{QV}$, na medida em que se tornam pouco evidentes. Apesar de tais dificuldades não afetarem diretamente a percepção subjetiva da saúde do idoso, elas precisam ser apreciadas pelos profissionais da área de saúde, com o intuito de contribuírem para os adiamentos de suas manifestações.

No que se refere à faceta "morte e morrer", o estudo de Frumi \& Celich $^{28}$ revelou que a maior preocupação dos idosos não está ligada ao evento em si, mas ao medo de sofrimento que possa anteceder o processo de morte. Além deste fator, a constatação dos idosos de que seus amigos e familiares estão sofrendo e morrendo, aumenta a percepção do evento como um processo doloroso, potencializando o sentimento de insegurança, tristeza e insatisfação.
Com relação aos altos índices de SV encontrados neste estudo, ainda são poucas as pesquisas que buscam verificar os níveis de SV em idosos (Sommerhalder ${ }^{29}$ ), porém Reker $^{30}$ e Krause $^{31}$ aludem para a possibilidade de que os idosos sejam um grupo com maior capacidade para apreender a existência com mais sentido e propósito do que adultos e pessoas mais jovens. Assim, ficariam mais realistas em relação à perseguição de metas e com menos probabilidades de sentirem frustração e vazio existencial. Para Recker, ${ }^{30}$ eles poderiam ter uma percepção mais fiel da realidade e de suas capacidades, tornando suas decisões mais acessíveis, realizáveis, compatíveis com a situação e a finalização efetiva da tarefa.

Quanto à DF, embora o processo de envelhecimento possa ser caracterizado por perdas funcionais, a capacidade funcional dos participantes da pesquisa apresentou índices positivos, ou seja, os dados sugerem que grande parte dos indivíduos, com 65 anos ou mais, está com a capacidade funcional preservada. Esse achado corrobora outros estudos de base populacional: ${ }^{32}$ por exemplo, Giacomin et al. ${ }^{32}$ constataram que $84 \%$ dos idosos participantes de seu estudo eram totalmente independentes para atividades de vida diária.

É preciso considerar que os resultados encontrados se devem, em parte, à natureza das tarefas investigadas pela MIF. Isto é, o instrumento avalia, com exceção de "locomoverse uma distância de 50 metros" e "subir e descer escadas", apenas atividades básicas de vida diária (ABVD) que correspondem à manutenção das funções mais elementares à sobrevivência do sujeito. Isso ocasiona menor sensibilidade do instrumento em demonstrar pequenas alterações na dependência funcional, tendo menos capacidade de perceber pequenas alterações da funcionalidade. Ou seja, o instrumento possivelmente não conseguiria discriminar idosos com baixa DF, diferenciando, por exemplo, apenas aqueles que necessitassem de muita ajuda para alimentar-se, vestir-se e tomar 
banho. Dessa maneira, sugerem-se para futuros estudos instrumentos que averiguem atividades instrumentais de vida diária (AIVD) mais complexas, tais como: tomar medicamentos, utilizar transporte, cuidar da casa, usar o telefone, entre outras.

Com relação à dimensão de locomoção, composta pelas AIVDs "subir e descer escadas" e "caminhar uma distância de 50 metros", observou-se que apresentou significativamente menores médias. Nessa perspectiva, Parahyba \& Simões ${ }^{33}$ apontam as dificuldades de locomoção, sobretudo as relacionadas a médias distâncias, como um indicador eficaz da perda funcional, na medida em que, hierarquicamente, são as AIVDs as primeiras a serem comprometidas.

Sentido de Vida como moderador do efeito da Dependência Funcional na Qualidade de Vida

Neste estudo, o SV parece ter operado como um recurso protetor da $\mathrm{QV}$ contra os aspectos da incapacidade funcional do idoso. Considerando que o WHOQOL-Old avalia a QV por meio da autoavaliação do indivíduo, acredita-se que os idosos com alto SV experimentem a incapacidade funcional como menos nociva à QV geral. Segundo McKnight \& Kashdan, ${ }^{34}$ o SV atua auxiliando a percepção de eventos, oferecendo uma sensação de significado existencial. Para os autores, o SV, por si só, não regula o comportamento, mas direciona os sujeitos para que, por meio da utilização de seus recursos psicossociais, possam superar os efeitos deletérios dos problemas de saúde. De acordo com Halama, ${ }^{35}$ o SV é capaz de ressignificar as situações de dificuldade, agindo como recurso apaziguador (buffer) capaz de modificar durante a etapa de avaliação, a percepção e/ou significado de eventos estressores.

Segundo Frankl, ${ }^{15,16}$ o SV é a principal força motivadora do ser humano; assim, toda dinâmica da vida estaria ligada à busca de sentido para sua existência. Frankl propõe que o SV tende a atuar como um efeito de tampão ou deslocamento das consequências deletérias das dificuldades no bem- estar do indivíduo. Dessa forma, a percepção de que o sofrimento tem um sentido, as dificuldades impostas pela incapacidade funcional poderiam ser encaradas como um desafio compreensivo e manejável. Frankl $1^{15,16}$ destaca que ninguém pode encontrar o SV por outra pessoa, mas pode facilitar o descobrimento, ao mostrar que é possível encontrá-lo até o último momento da vida. Sempre se poderá mudar de atitude diante de um evento, mesmo que este não possa ser alterado. Para autores como Ho, Cheung \& Cheung ${ }^{13}$ e Schulenberg \& Melton, ${ }^{22}$ o SV é um componente fundamental para o processo de resiliência, a qual seria a operacionalização de algumas das consequências do SV.

Por fim, o estudo apresenta algumas limitações a serem consideradas. A primeira se refere ao método transversal de caráter eminentemente correlacional, não sendo possível realizar inferências causais entre as variáveis. A segunda se deve ao reduzido tamanho da amostra, pois se perderam alguns participantes desde o primeiro estudo, descaracterizando a representatividade amostral do estudo Rede Fibra; e uma terceira limitação alude para a ausência de utilização de outras medidas neuropsicológicas, capazes de assegurar ou dar maior fiabilidade na avaliação e distinção de idosos com comprometimento cognitivo ou com demência.

\section{CONSIDERAÇOEES FINAIS}

Este estudo objetivou avaliar os índices de sentido de vida (SV), qualidade de vida (QV) e dependência funcional (DF) de idosos, e observar se o SV poderia atuar como recurso protetor capaz de diminuir os efeitos da DF na QV. Os resultados indicaram, para a maioria da amostra, baixos índices de DF e bons índices de SV e QV. Não obstante, para aqueles idosos que apresentaram altos índices de SV, a DF não explicou significativamente a $\mathrm{QV}$, sugerindo um afeito moderador do SV.

Os resultados deste estudo chamam a atenção para a importância de se pensar formas de intervenção que possam ser baseadas em 
trabalhos multidisciplinares, que incluam tanto profissionais que atuem no sentido de diminuir a DF e também profissionais que possam facilitar o desenvolvimento do SV. Assim, intervenções baseadas nos pressupostos da logoterapia poderiam ser implementadas com o intuito de melhoria na QV de idosos com alta DF.

Será fundamental seguir investigando neste âmbito da qualidade de vida em idosos. A identificação de fatores que a promovam, mesmo diante de eventos estressantes que parecem ser inevitáveis e inerentes ao processo de envelhecimento, deveria constituir uma agenda de pesquisa. Parece pouco produtivo

\section{REFERÊNCIAS}

1. Organização Mundial da Saúde. Envelhecimento ativo: uma política de saúde. Brasília: OPS; 2005.

2. Instituto Brasileiro de Geografia e Estatística. Censo Demográfico 2010: sinopse do censo e resultados preliminares do Universo. 2011 [acesso em 12 jan 2011]. Disponível em: http://www.ibge. gov.br/home/presidencia/noticias/imprensa/ ppts/0000000402.pdf

3. Yang Y, George LK. Functional disability, disability transitions, and depressive symptoms in late life. J Aging Health 2005;17(3):263-92.

4. Nunes DP, Nakatani AYK, Silveira EA, Bachion MM, Souza MR. Capacidade funcional, condições socioeconômicas e de saúde de idosos atendidos por equipes de Saúde da Família de Goiânia (GO, Brasil). Ciênc Saúde Coletiva 2010;15(6):2887-98.

5. Alves LC, Leite IC, Machado CJ. Fatores associados à incapacidade funcional dos idosos no Brasil: análise multinível. Rev Saúde Pública 2010;44(3):468-78.

6. Marra TA, Pereira LSM, Faria CDCM, Pereira DS, Martins MAA, Tirado MGA. Avaliação das atividades de vida diária de idosos com diferentes níveis de demência. Rev Bras Fisioter 2007;11(4):267-72.

7. Vivan AS, Argimon IIL. Estratégias de enfrentamento, dificuldades funcionais e fatores associados em idosos institucionalizados. Cad Saúde Pública 2009;25(2):436-44.

8. Fortes-Burgos ACG, Neri AL, Cupertino APFB. Eventos de vida estressantes entre idosos brasileiros residentes na comunidade. Estud Psicol (Natal) 2009;14(1):69-75. dirigir o olhar apenas para os fatores de risco da qualidade de vida.

Por fim, o PIL-Test 12, enquanto medida de avaliação de sentido de vida, não discrimina as fontes de sentido. Para futuras investigações, seria enriquecedor avaliar quais aspectos estão relacionados com a presença ou ausência de sentido de vida na população estudada, visto que esta informação facilitaria a execução de intervenções que tenham como objetivo trabalhar aspectos humanísticos e existenciais dos sujeitos. Espera-se que estudos posteriores possam replicar ou refutar os resultados preliminares apresentados nesta investigação.

9. Paschoal SMP. Autonomia e independência. In: Papaléo-Netto M, organizador. Gerontologia: a velhice e o envelhecimento em visão globalizada. São Paulo: Atheneu; 2002. p. 311-23.

10. Fleck MPA, Louzada S, Xavier M, Chachamovich E, Vieira G, Santos L, et al. Aplicação da versão em português do instrumento abreviado de avaliação da qualidade de vida "WHOQOLbref". Rev Saúde Pública 2000;34(2):178-83.

11. Margis R, Picon P, Cosner AF, Silveira RO. Relação entre estressores, estresse e ansiedade. Rev Psiquiatr Rio Gd Sul 2003;25(1):65-74.

12. Fillion L, Dupuis R, Tremblay I, De Grâce GR, Breitbart W. Enhancing meaning in palliative care practice: a meaning-centered intervention to promote job satisfaction. Palliat Support Care 2006;4(4):333-44.

13. Man YH, Fanny MC, Shu FC. The role of meaning in life and optimism in promoting wellbeing. Personality and Individual Differences 2010;48(5):658-63.

14. Melton AMA, Schulenberg SE. On the measurement of meaning: Logotherapy's Empirical Contributions to Humanistic Psychology. The Humanistic Psychologist 2008;36:31-44.

15. Frankl VE. Psicoterapia e sentido de vida: fundamentos de Logoterapia e análise existencial. 3. ed. São Paulo: Quadrante; 1989.

16. Frankl VE. Em busca de sentido: um psicólogo no campo de concentração. 19. ed. Petrópolis: Vozes; 2004. 
17. Folstein MF, Folstein SE, McHugh PR. "Mini-mental state". A practical method for grading the cognitive status of patients for the clinician. J Psychiatr Res 1975;12(3):189-98.

18. Brucki SMD, Nitrini R, Caramelli P, Bertolucci PHF, Okamoto IH. Sugestões para o uso do mini-exame do estado mental no Brasil. Arq Neuro-Psiquiatr 2003;61(3B):777-81.

19. Fleck MP, Chachamovich E, Trentini C. Desenvolvimento e validação da versão em Português do módulo WHOQOL-OLD. Rev Saúde Pública 2006;40(5):785-791.

20. Chachamovich E. Qualidade de Vida em idosos: desenvolvimento e aplicação do módulo WHOQOL-OLD e teste do desempenho do instrumento WHOQOL-BREF em uma população idosa brasileira [dissertação de Mestrado]. Porto Alegre: Programa de pós-graduação em Ciências Médicas da Universidade Federal do Rio Grande do Sul; 2005.

21. Crumbaugh JH, Maholich LT. The psychometric approach to Frankl's concept of noogênica neurosis. Journal of Clinical Psychology 1964;20:200-7.

22. Schulenberg SE, Melton AMA. A confirmatory factor-analytic evaluation of the purpose in life test: preliminary psychometric support for a replicable two-factor model. J Happiness Stud 2010;11:95-111.

23. Aquino TAA. Atitudes e intenções de cometer suicídio: seus correlatos existenciais e normativos [tese de Doutorado]. João Pessoa: Departamento de Psicologia da Universidade Federal da Paraíba; 2009.

24. Pollak N, Rheault W, Stoecker JL. Reliability and validity of the FIM for persons aged 80 years and above from a multilevel continuing care retirement community. Arch Phys Med Rehabil 1996;77(10):1056-61.
25. Riberto M, Miyazaki MH, Jorge Filho D, Sakamoto H, Battistella LR. Reprodutibilidade da versão brasileira da medida de independência funcional. Acta Fisiátrica 2001;8(1):45-52.

26. Baron RM, Kenny DA. The moderator-mediator variable distinction in social psychological research: conceptual, strategic, and statistical considerations. J Pers Soc Psychol 1986;51(6):1173-82.

27. Faller JW, Melo WA, Versa GLGS, Marcon SS. Qualidade de vida de idosos cadastrados na estratégia saúde da família de Foz do Iguaçu-PR. Esc Anna Nery Rev Enferm 2010;14(4):803-10.

28. Frumi C, Celich KLS. O olhar do idoso frente ao envelhecimento e à morte. RBCEH 2006;3:92-100.

29. Sommerhalder C. Sentido de vida na fase adulta e velhice. Psicol Reflex Crit 2010;23(2):270-7.

30. Reker GT. Manual of the Life Attitude ProfileRevised (LAP-R). Peterborough, Canada: Student Psychologists Press; 1992.

31. Krause N. Evaluating the stress-buffering function of meaning in life among older people. J Aging Health 2007;5(19):792-812.

32. Giacomin KC, Peixoto SV, Uchoa E, Lima-Costa MF. Estudo de base populacional dos fatores associados à incapacidade funcional entre idosos na Região Metropolitana de Belo Horizonte, Minas Gerais, Brasil. Cad Saúde Pública 2008;24(6):1260-70.

33. Parahyba MI, Simões CCS. A prevalência de incapacidade funcional em idosos no Brasil. Ciênc Saúde Coletiva 2006;11(4):967-74.

34. McKnight PE, Kashdan TB. Purpose in life as a system that creates and sustains health and wellbeing: An integrative, testable theory. Review of General Psychology 2009;13(3):242-51.

35. Halama P, Bakosová K. Meaning in life as a moderator of the relationship between perceived stress and coping. Studia Psychologica 2009;51(2):143-8. 